\title{
HERF: A Hybrid Energy Efficient Routing using a Fuzzy Method in Wireless Sensor Networks
}

\author{
Zohre. Arabi ${ }^{1}$ and Yaghoub Khodaei ${ }^{2}$ \\ ${ }^{1}$ Young Researchers Club, of Tabriz ,Islamic Azad University, Tabriz Branch \\ Tabriz, IRAN \\ Zohre.arabiegmail.com \\ ${ }^{2}$ Jobholder of Bank Meli Iran, Tabriz, IRAN \\ Yaghoub.khodaei@gmail.com
}

\begin{abstract}
Wireless Sensor Network (WSN) is one of the major research areas in computer network field today.Data dissemination is an important task performed by wireless sensor networks. The routing algorithms of this network depend on a number of factors such as application areas, usage condition, power, aggregation parameters. With respect to these factors, different algorithms are recommended. One of the most important features of routing algorithms is their flexibility and ability to self-organize themselves according to such parameters. The existence of flexibility in routing protocols can satisfy calls for on demand and table driven methods. Switching between these two methods would be impossible except by compatibility between nodes' and switcher. Energy is another significant factor in wireless sensor networks due to limited battery power and their exchangeability. To arrive at a network with mentioned features, we have proposed an algorithm for hybrid energy efficient routing in wireless sensor networks which uses two algorithms, i.e. EF-Tree (Earliest-First Tree) and SID (Source-Initiated Dissemination) to disseminate data, and employs a fuzzy method to choose cluster head, and to switch between two methods, i.e. SID and EF-Tree. In this routing, the whole network is clustered and the appropriate clusterhead is selected according to fuzzy variables. Then, analyzing the changes in fuzzy variables and If fuzzy, then rule, one routing in EF-Tree or SID is chosen for information transmission. The results of simulations indicate that HERF has improved energy efficiency.
\end{abstract}

\section{KEYWORDS}

Data Dissemination, Wireless Network, Fuzzy Routing Protocols, Virus, SID \& EF-Tree

\section{INTRODUCTION}

WSNs (wireless sensor networks) present a new generation of real-time embedded system for a wide variety of applications[1].However WSNs have limited computation, energy, and memory resource. [1] These small, inexpensive sensors are able to provide wireless internetworks communication and perform tasks such as control, observing inaccessible environments, medical applications, intrusion detection, surveillance tactical, etc [2]. In addition, wireless sensor networks are self-organizing and adapt themselves with changes due to node damage, node movement across the network [3]. Designing the WSNs is very difficult because the sensor nodes have limited computation capability, limited power and small memory size [4][5]. WSN aims at aggregating data from environment and sending it to Base Station (BS) in order to process and compute, while they are conducted through different ways. In a hybrid method, various routing methods are employed to send information, and since there are different routing 
algorithms due to different applications and environmental features for data dissemination, some algorithms are selected that can complete each other. Two algorithms which are selected in Multi[7] for hybrid are SID algorithm that is a type of proactive algorithms and a branch of reactive routing algorithms, and routing EF-Tree algorithm which is used with respect to the number of network events. The analysis of hybrid routing algorithm indicates that the hybrid usage of these two decreases the network's concentration and increases the network lifetime [7]. Furthermore, one kind of the famous routing algorithms to achieve the energy efficiency is cluster routing[8][9]. In cluster routing, the network clusters are divided into clusters, and for each cluster one cluster-head is selected, and this very cluster is responsible for data aggregation from nodes inside the cluster and concentrating them and sending concentrated information to BS [9]. In clustering method, as communication to BS is limited to cluster-heads, the overhead due to communication decreases. Although employing clustering technique lowers consumption energy, it raises problems such as cluster selection and cluster-head energy consumption. LEACH [10] is an algorithm which uses local clustering based on probabilities. In LEACH all sensor nodes have the same chance to become cluster head, i.e. a node in the cluster margin and a node in the center, or a node with little remaining energy and a node with higher energy have similar chance to be selected. As such, there is a possibility for selecting inappropriate cluster head, or inefficiency of energy in LEACH. There are other fuzzy-based methods for selection of cluster-heads. Taking variables such as concentration and energy, the best way for cluster-head selection is making fuzzy and concentrated these variables using if fuzzy then rule which lowers BS computational load due to its local computation and also increase the network lifetime [1]. In general, a network should be self organizing so that it can adjust itself for doing tasks in different conditions [3]. The proposed resolution here is using energy efficient hybrid routing based on fuzzy method that adjust itself with current traffic in the network. The name of the suggested method implies that it has a set of features of three previous methods, i.e. SID, EFTree, Fuzzy method which are performed alternatively and are switched between routings due to conditions. This is a novel method for data dissemination in WSNs.

The remainder of the paper is organized as follows: section 2 presents related work, section 3 outlines proposed routing algorithm, its simulation and efficiency analysis. Section 4 provides the conclusions and suggestions for further work.

\section{Related Work}

Several communication protocols have been proposed to realize power-efficient communication in the WSN [11-17].The Directed Diffusion protocol proposed in [18-19] is data centric in that all communication is for named data. All nodes in a directed diffusion-based network are application aware. This enables diffusion to achieve energy savings by selecting empirically good paths and by caching and processing data in-network. This protocol is not usable for the applications that require continuous data delivery to the sink such as environmental monitoring. In addition, it generates more traffic control in the network.The method proposed in Multi protocol [7] permits wireless sensor networks to use independent routing methods but compatible with different traffic conditions to raise energy efficiency. This is done by two routing algorithms, i.e. SID and EF-Tree.

The switch between these two routing algorithms is conducted based on comparison of revealed events with determined limit for the network by the sensor nodes. The most important feature of these two algorithms is their high changeability in routing. EF-Tree and SID are different as SID is a reactive algorithm that does not require an initial substructure for data dissemination. The rout discovery is done after event occurrence, and once the event is reported, there is no need to maintain and keep the rout up-dated. As a result, the overhead of routing is low. This type of routing is more appropriate for environments with few number of transactions. 
EF-Tree algorithm uses proactive routing method for connecting nodes and updates its routing tables periodically using control messages, and it welcomes probable changes too. Such routing is more efficient for environments with many transactions and events [7].

In protocol Multi [7] the time of switch between two routings is determined based on the limit for the number of events. The network starts its work with SID, and it switches to EF-Tree as the number of events that reach limit increases. When the number of events is decreased to lower than the limit, SID is used again and so on. Although this protocol somewhat increases the network lifetime, it is not an appropriate protocol since the nature of switch is vague and it is not clear which node(s) is responsible for determining events and comparing the limit.

SHARP [20] is a routing protocol for ad hoc networks that founds an equilibrium between reactive and proactive protocols adapting the degree of how routing information is propagated in the network. Clustering such is another approach for arriving at energy efficiency in communication between sensor nodes.

Different methods are presented for clustering and cluster- head selection, and LEACH is one example. It employs probability model for nodes' clustering and clusters selection. In this method, since no attention is given to remaining energy of node and node's location at the cluster, there may be extra consumption energy. In some cases, however, a proper cluster-head may be selected accidently.Gupta [1] is another method which has analyzed the application of fuzzy variables to make the network life time efficient. In this method, the cluster selection is performed based on energy fuzzy variables, centrality, and concentration. The weak point in Gupta is its concentration, as a heavy load is imposed to BS, since BS has to decide whether nodes are cluster-heads.

CHEF[21] uses techniques for clustering similar to those of Gupta[1]. In CHEF, however, the work load is decreased These works are performed locally inside the clusters, i.e. all nodes' information is no longer sent to BS, but decision on cluster-head is made inside the cluster considering variables as energy, concentration and centrality. As such consumption energy is considerably decreased.

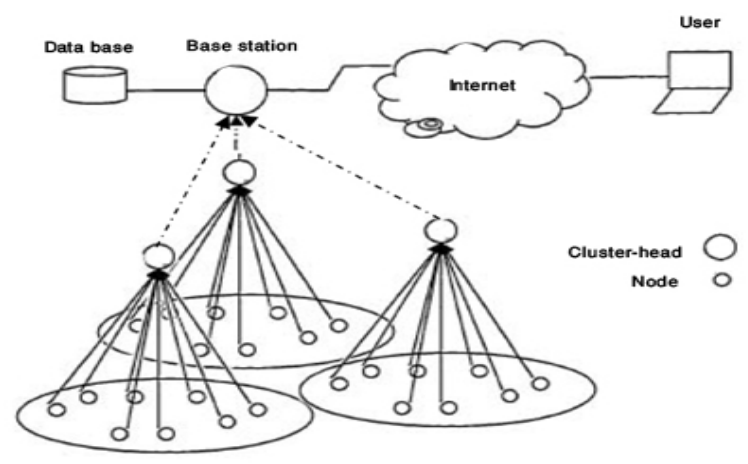

Figure 1. Main architecture of WSN

\section{Proposed Algorithm}

This section deals with HERF algorithm. This routing algorithm is made from some steps for data dissemination: 


\section{1. cluster-head selection}

1) Fist Phase: Determining initial chance

Clustering is used in HERF. It is assumed that all nodes have similar initial energy. The main architecture of WSN is represented in Figure 1.

$X$ : remaining energy

$$
\gamma=\frac{x}{\operatorname{Red}}
$$

Red: initial energy

$\gamma$ represents initial chance of the node to become cluster-head, and its value is distributed across nodes inside the cluster along with the candidate message. If the value of $\gamma$ is lower than $0 / 1$, that node no longer sends candidate message to other nodes. All nodes inside the cluster wait for a given time to receive candidate messages from other cluster nodes, and finally comparing with $\gamma$ values received from candidate messages and $\gamma$ itself, nodes with highest values are determined as those with highest chance for clustering.

2) Second Phase-Implementation of fuzzy if then
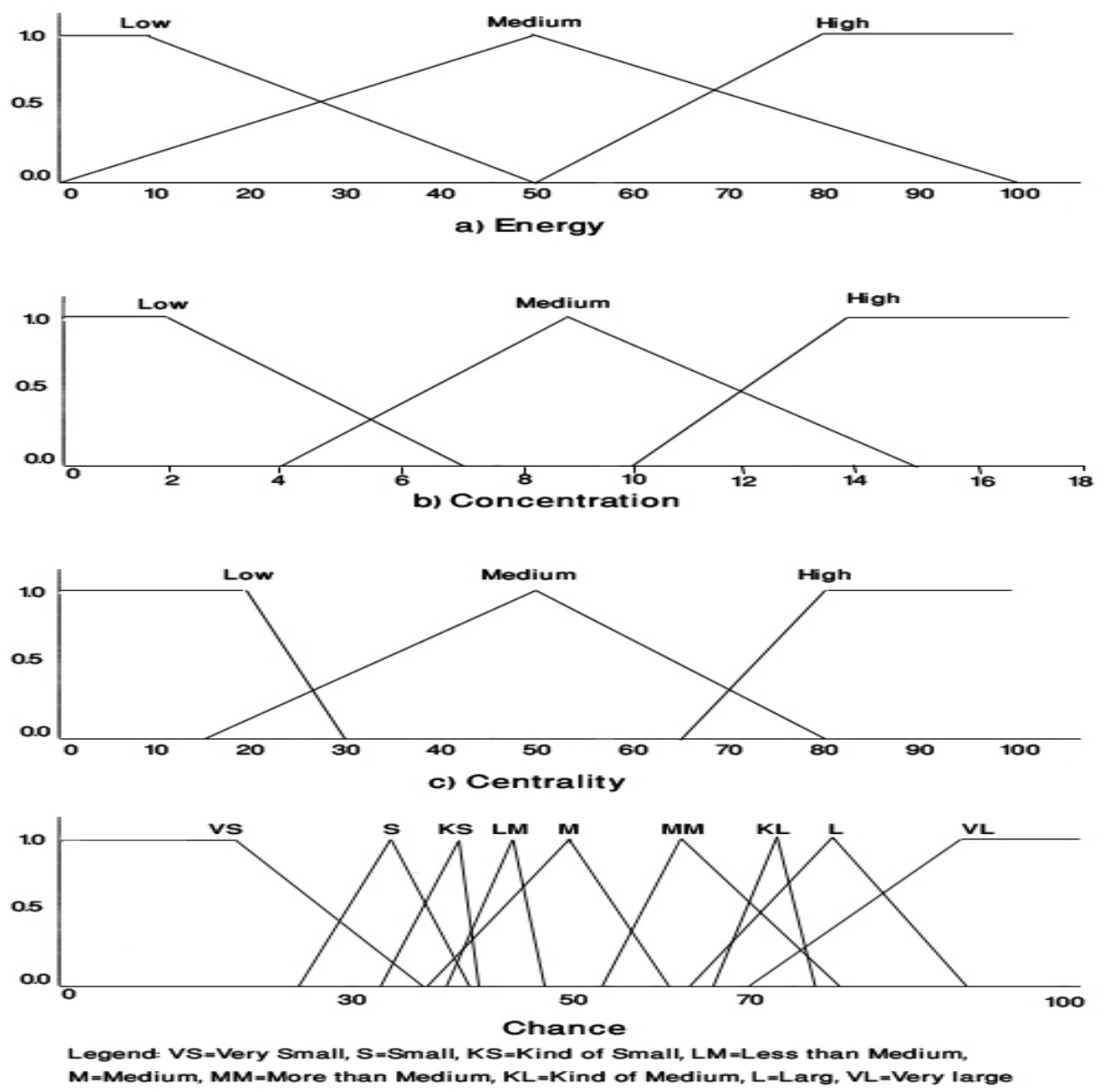

Figure 2. Fuzzy set for fuzzy variables used in cluster-head selection 
In this step fuzzy if then rule is selected to select the best cluster-head in the first phase. To do so, fuzzy variables Node Energy, Node concentration, and Node centrality are used. They are defined as below:

Node Energy: ratio of node remaining energy to node initial energy $(\gamma)$

Node concentration: number of nodes present in the vicinity

Node centrality: a value which classifies the nodes based on central the node is to the cluster.

Table 1. a) Fuzzy if then rule for cluster-head selection, b) Fuzzy if then rule for route selection

\begin{tabular}{|c|c|c|c|c|c|c|c|}
\hline \multicolumn{4}{|l|}{ a) } & \multirow[t]{2}{*}{ b) } & & & \\
\hline Energy & \multicolumn{2}{|c|}{ Density } & $\begin{array}{l}\text { ontrality } \\
\text { grade }\end{array}$ & & $\begin{array}{l}\text { Type of } \\
\text { routing }\end{array}$ & Energy & Event \\
\hline Very Small & Low & Low & Low & & SID & Low & Low \\
\hline Small & Low & Low & High & & SID & Medium & Low \\
\hline Small & Low & Low & Medium & & SID & High & Low \\
\hline Small & Low & Medium & Medium & & SID & Low & Medium \\
\hline Small & Low & Medium & Low & & EF-Tree & Medium & Medium \\
\hline Small & Low & High & Low & & EF-Tree & High & Medium \\
\hline Kind of Small & Low & Medium & High & & SID & Low & High \\
\hline Less than Medium & Low & High & High & & EF-Tree & Medium & High \\
\hline Less than Medium & Medium & Low & Low & & EF-Tree & High & High \\
\hline Less than Medium & Low & High & Medium & & & & \\
\hline Medium & Medium & Low & Medium & & & & \\
\hline Medium & Medium & Medium & Low & & & & \\
\hline Medium & High & Medium & Low & & & & \\
\hline Medium & High & High & Low & & & & \\
\hline More than Medium & Medium & Medium & Medium & & & & \\
\hline More than Medium & Medium & Medium & High & & & & \\
\hline More than Medium & Medium & High & Low & & & & \\
\hline More than Medium & Medium & Low & High & & & & \\
\hline More than Medium & High & Low & Medium & & & & \\
\hline More than Medium & High & Low & Low & & & & \\
\hline Kind of Large & High & High & Medium & & & & \\
\hline Kind of Large & High & Medium & Medium & & & & \\
\hline Kind of Large & High & Low & High & & & & \\
\hline Kind of Large & Medium & High & Medium & & & & \\
\hline Large & Medium & High & high & & & & \\
\hline Large & High & Medium & High & & & & \\
\hline Very Large & High & High & High & & & & \\
\hline
\end{tabular}

Considering the variables that have been fuzzy and fuzzy if then rule represented in Table 1(a), the node with highest chance to be selected as cluster-head is chosen. As shown in Table 1, it can be concluded that energy has priority over concentration, and concentration has priority over centrality. For example, a node with high energy and concentration and average centrality 
has more chance over a node with average energy and concentration and high centrality. The details of Fuzzy set for energy, concentration, and centrality are illustrated in Figure 2. The node chosen as cluster-head sends Join Message to all nodes of the same cluster, therefore, cluster-

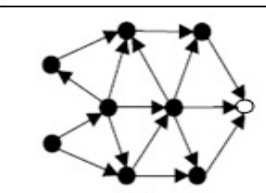

(a)

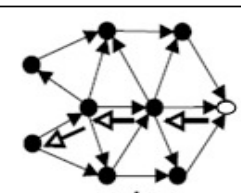

(b)

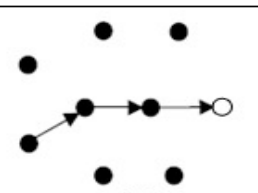

(c)

I.EF-Tree algorithm

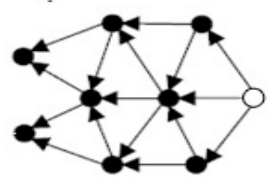

(a)

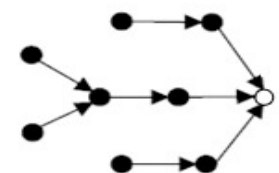

(b)

\section{II.SID algorithm}
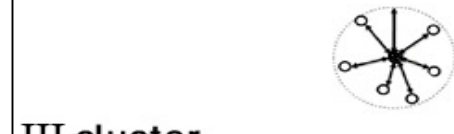

Base station $\bigcirc$ Cluster-Head

\section{III.cluster}

Sensor nod

Figure 3. I)EF-Tree algorithm:(a)Tree building, (b) Data dissemination towards the base station. II)SID algorithm:(a) Data diffusion, (b) Requisition messages, (c) Data dissemination towards the base station III)Cluster internal structure.

head is introduced to all nodes.

\section{2. data dissemination from cluster-head to BS}

There are different algorithms for data dissemination in wireless sensor networks which can be classified into two routing approaches: centralized routing [22,23], and distributed routing $[24,26]$.

The distributed approach is itself classified into two groups: reactive approach and proactive approach.

In the proactive approach, routing topology is created prior to data transmissions from mobile nodes. The routing information is then dynamically updated according to changes of the network topology.

In contrast, the reactive routing approach assumes no existing routing state in the network prior to data transmission from the particular station. Upon arrival of a first data packet the node enters a route discovery phase in which it announces the request for the particular destination address to the network.

In reactive routing the routing information is maintained in the network only for the period of activity of the particular session[21].SID and EF-Tree algorithms have been described earlier in section 2, and it is explained that SID follows reactive approach and EF-Tree takes proactive approach [Figure 3]. 
Employing these two algorithms and taking fuzzy variables of event and energy into account, and also using fuzzy if then rule introduced in Table 1(b), the type of routing for next period is determined.

Event: number of reported events to BS during a given period

Energy: energy average of cluster-heads sending message to BS during a given period

The details of fuzzy set of these variables are illustrated in Figure 4.
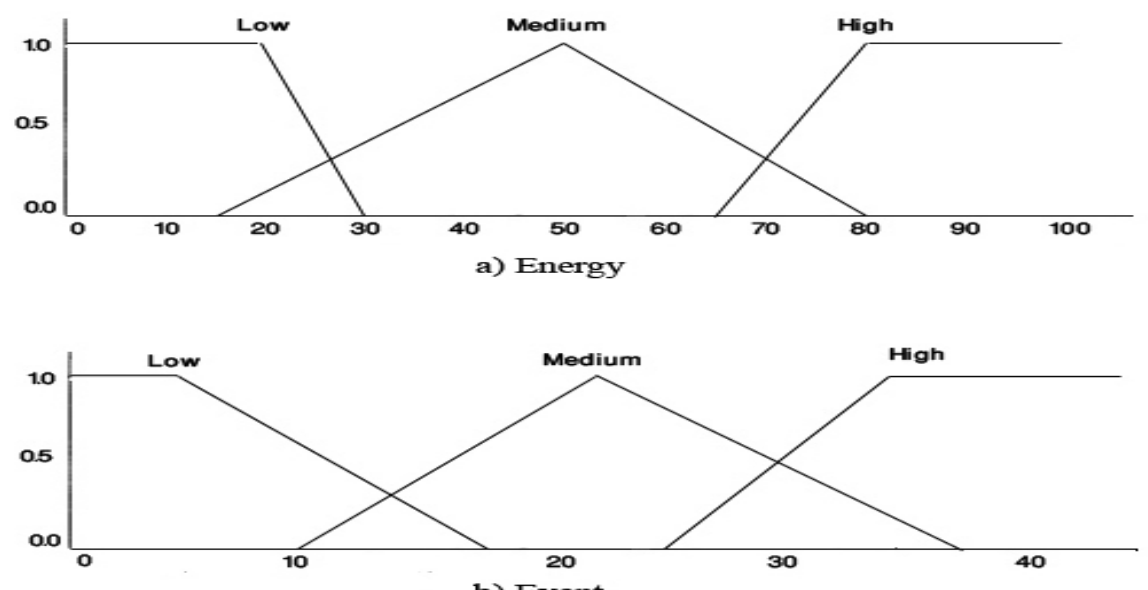

b) Event

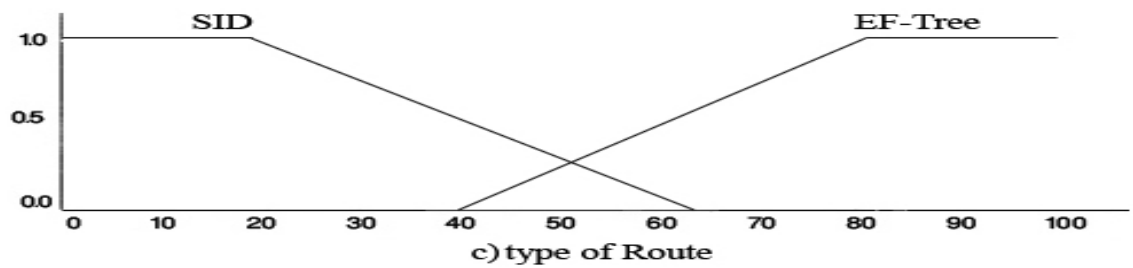

Figure 4. Fuzzy set for fuzzy variables used in routing selection

\section{Simulations and performance analysis}

To evaluate the proposed method, its efficiency is compared with Multi[7] and CHEF[21] considering different aspects. For simulation, simulator MATLAB and a network with 100 nodes which are randomly scattered in $100 \mathrm{~m} \times 100 \mathrm{~m}$ square. The required parameters is: initial power $=0.5 \mathrm{~J}$, Transmitter Electronics $=50 \mathrm{~nJ} / \mathrm{bit}$, Receiver Electronics $=50 \mathrm{~nJ} / \mathrm{bit}$.

In HERF, using fuzzy logic for selecting cluster-head causes selection of the node with the longest life time as the cluster-head.

Using $\gamma$ to determine the initial chance is another advantage of method and it decreases the number of comparisons in the second phase. Selection of the value $0 / 1$ as the limit value is based on simulation results. 
Simulation shows that the node with energy value $\gamma=0 / 1$ and lower can provide required energy just for sending join message, and it becomes inactive then. As a result, adding a node with $\gamma \leq 0 / 1$ increases work load of cluster-head.

In addition, selecting only one cluster-head for distance $r$ is another advantage of this method ( $r$ equals the maximum distance between two nodes inside cluster divided to 2).

Comparison of with Gupta[1] shows that in the former decision and comparison is made in BS, all comparisons and decisions for selecting cluster-heads is made inside the cluster, and no load

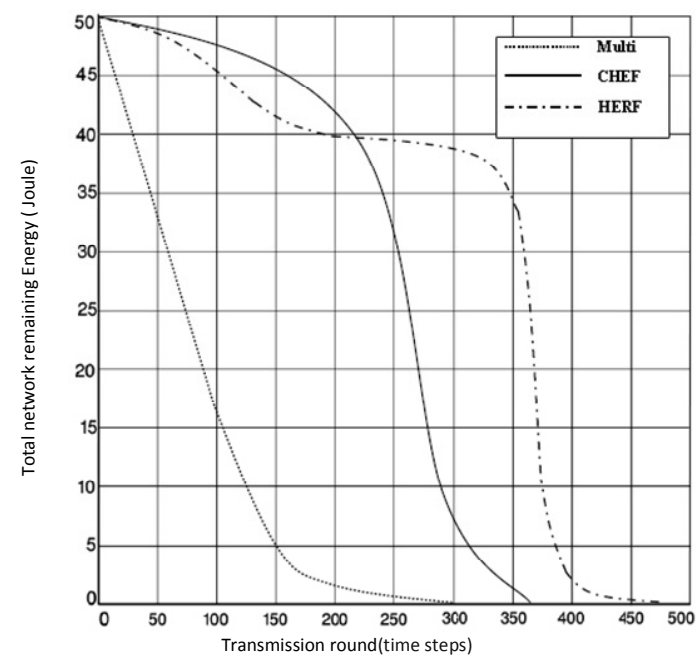

a) Network total residual energy variation versus

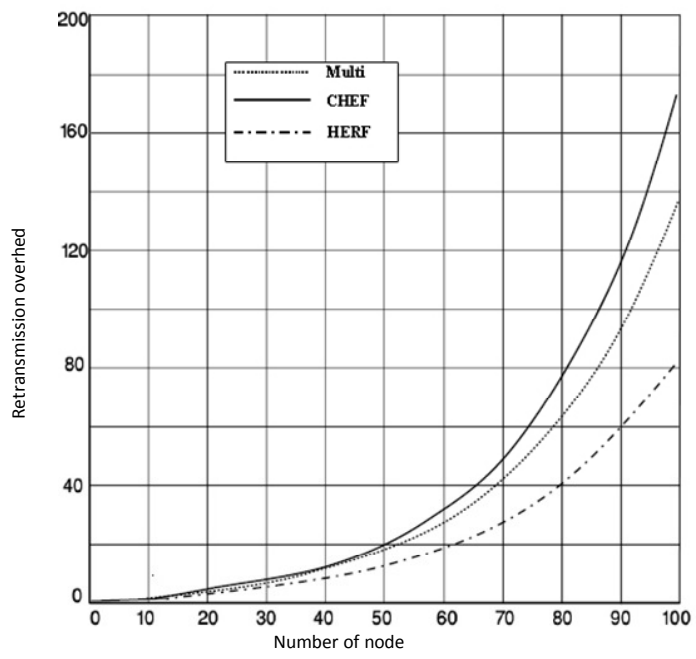

b) Network overhead

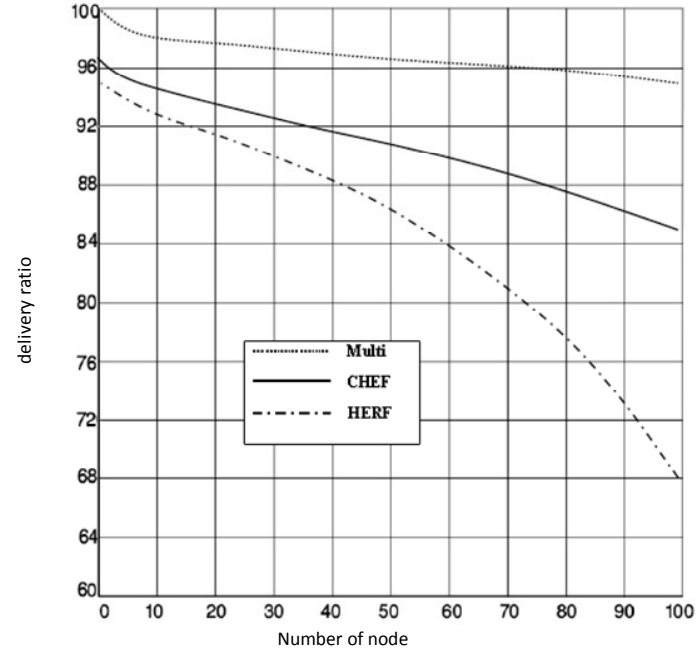

c) delivery ratio

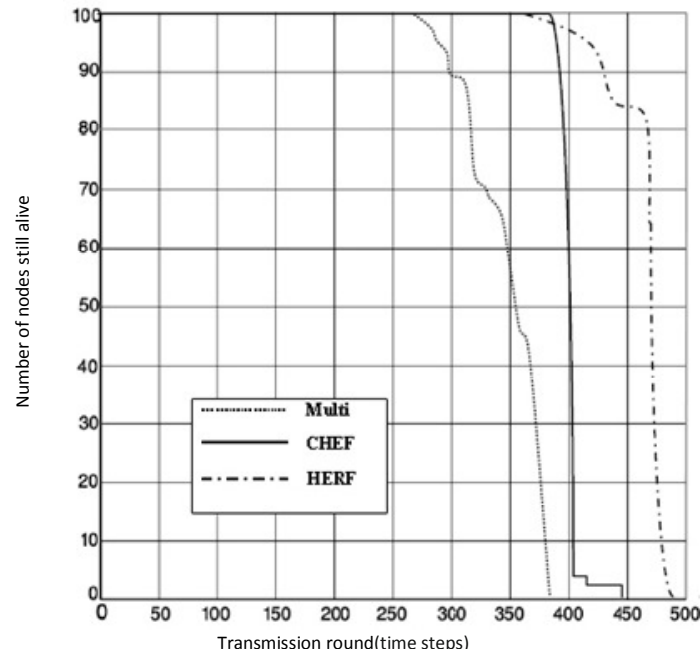

d) Network lifetime versus transmission round

Figure 5. simulation result 
is added to BS. Furthermore, is far from many weak points evidenced in LEACH[10] that employs probability model for selecting cluster-head.

CHEF [21] has presented an algorithm for selecting cluster based on fuzzy logic, but it is lower efficiency over due to calculating initial chance based on probabilities.

Multi is another algorithm for routing which follows hybrid routing. Here, there is vagueness on determining switch parameters and the manner of switch., however, has removed such problems.

The method proposed in this study is a new work on routing in wireless sensor networks which is based on fuzzy and hybrid routing methods for higher flexibility on the network, increase of energy efficiency, and increase of the network life time. Our result illustrated by Figures5.

In this study a new method is introduced for selecting cluster-head and data dissemination using fuzzy logic and hybrid routing, and considering different conditions governing on WSN.

The simulation results show that the proposed system has increased energy efficiency using fuzzy variables and appropriate decision making based on existing conditions of the network. A further direction of this study would be using other fuzzy variables in selecting cluster-head and switching between routings.

\section{ACKNOWLEDGEMENTS}

I would like to thank my thesis supervisor, Dr Amirmasoud Rahmani for his encouragement and guidance.

\section{REFERENCES}

[1] I. Gupta, D. Riordan and S. Sampalli, "Cluster-head Election using Fuzzy Logic for Wireless Sensor Networks", ommunication Networks and Services Rearch Conference, pp.255-260, May 2005.

[2] I.F. Akyldiz, W. Su, Y. Sankarasubramaniam, E. Cayirci., "A Survey on Sensor Networks", IEEE Wireless Communication Magazine, Vol. 40, no. 8, pp. 102-116, 2002.

[3] Premaratne, K., Zhang, J., \& Dogruel, "Location information-aided task- oriented selforganization of ad hoc sensor systems”. Sensors Journal, 4(1), 85-95. M 2004.

[4] J.N. Alkaraki and A.E. Kamal, "Routing Techniques in Wireless Sensor Networks: A Survey", IEEE Wireless Communication, pp. 6-23, December 2004.

[5] K. Rerkrai and Ch. Jardak, "Demo Abstract: Survivable and Scalable WSN Solution for Environmental Monitoring in Harsh Conditions”,2005 IEEE.

[6] Piyakul Tillapart, Sanguan Thammarojsakul, Thanachai Thumthawatworn, Pratit Santiprabhob. "An Approach to Hybrid Clustering and Routing in Wireless Sensor Networks",2005 IEEE

[7] C. M. Figueiredo, E. F. Nakamura, and A. A. Loureiro. Multi: A hybrid adaptive dissemination protocol for wireless sensor networks. In proceedings of Algorithmic Aspects of Wireless Sensor Networks, ALGOSENSORS 2004, Turku, Finland, pages 171-186. Springer-Verlag 2004 .

[8] A. Manjeshwar and D.P. Agarwal, "TEEN: a Routing Protocol for Enhanced Efficiency in Wireless Sensor Networks”, Parallel and Distributed Processing Symposium,pp. 2009-2015, April 2001.

[9] S. Lindsey and C. Raghavendra, "PEGASIS: Power-Efficient Gathering in Sensor Information Systems", IEEE Aerospace Conference Proceedings , vol. 3, pp. 1125-30, 2002. 
International Journal of Distributed and Parallel systems (IJDPS) Vol.1, No.1, September 2010

[10] W.R. Heinzelman, A. Chandarkasan, and H. Balakrishnan, "Energy-Efficient Communication Protocol for Wireless Microsensor Networks", Proceedings of the 33rd Hawaii International Conference on System Sciences, 2000.

[11] Akyildiz, ., Su, W., Sankarasubramaniam, Y., \& Cayirci, E. "A survey on sensor networks", IEEE Communications Magazine, 40(8), 102-114. 2002.

[12] Kahn, J. M., Katz, R. H., \& Pister, K. S. J. "Next century challenges : Mobile networking for smart ", The Proceedings of 5th Annual ACM/IEEE International Conference on Mobile Computing and Networking (MobiCom 99) (pp. 271-278). 1999.

[13] Pottie, G. J., \& Kaiser, W. J. "Wireless integrated network sensor”, Communications of the ACM, 43(5), 51-58. 2000

[14] Tilak, S., Abu-Ghazalah, N.B., \& Heinzelman, W. "A taxonomy of wireless microsensor network models", ACM Mobile Computing and Communications Review (MC2R), 6(2), 2836. 2002.

[15] Efthymiou C, Nikoletseas S, Rolim J. "Energy Balanced Data Propagation in Wireless Sensor Networks[C] ",18th International parallel and Distributed Processing Symposium, Santa Fe: Kluwer Academic Publishers Hingham, 2004: 225-232.

[16] Zhang Jun, Zhao Erdun, Zhang Qiang. “Energy-Balanced Solution for Cluster-Based Wireless Sensor Networks with Mixed Communication Modes[C] ”,International Workshop Cross Layer Design. New York: IEEE Press, 2007: 29-32.

[17] Liu Xiangwen, Hou Huifeng, Yu Hongyi. LEBR: A Localized

[18] Intanagonwiwat, ., Govindan, R., \& Estrin, D. “Directed diffusion: A scalable and robust communication paradigm for sensor networks", The Proceedings of the 6th Annual ACM/IEEE International Conference on Mobile Computing and Networking (MobiCom'00) Boston, MA. 2000.

[19] Estrin, D., Govindan, R., Heidemann, J., \& Kumar, S. "Next century challenges: Scalable coordination in sensor networks", The Proceedings of the 5th Annual ACM/IEEE International Conference on Mobile Computing and Networking (MobiCom 99) (pp. 263-270).1999.

[20] Ramasubramanian, V., Haas, Z., Sirer, E. "SHARP: A hybrid adaptive routing protocol for mobile ad hoc networks", In: Proceedings of the 4th ACM Interational Symposium on Mobile Ad Hoc Networking and Computing (MobiHoc '03). (2003) 303-314.

[21] Kim, J, S. Park, Y. Han, T. Chung, "CHEF: Cluster Head Election mechanism using Fuzzylogic in Wireless Sensor Networks", Advanced Communication Technology ICACT 2008, pp. 654-659, 17-20 Feb. 2008.

[22] Deng, J., Han, R., Mishra, S. "INSENS: Intrusion-tolerant routing in wireless sensor networks", In: Department of Computer Science, University of Colorado, no. CU-CS-939-02, Boston, MA (2002)

[23] Li, Q., Aslam, J., Rus, D. “ Hierarchical power-aware routing in sensor networks”, In: Proc. DIMACS Workshop on Pervasive Networking (May 2001)

[24] Hill, J., Szewczyk, R., Woo, A., Hollar, S., Culler, D., Pister , K. “ System architecture directions for networked sensors", In: Proceedings of the International Conference on Architectural Support for Programming Languages and Operating Systems (ASPLOS) (2000) 
International Journal of Distributed and Parallel systems (IJDPS) Vol.1, No.1, September 2010

[25] Perkins, C., Belding-Royer, E., Das, S. “ Ad hoc On-Demand Distance Vector (AODV)

Routing”, RFC 3561 (Experimental) (July 2003), [Online]. Available http://www.ietf.org/rfc/rfc3561.txt

[26] Perkins, C.E., Bhagwat, P. " Highly dynamic destination-sequenced distance-vector routing (DSDV) for mobile computers”, In: SIGCOMM, pp. 234-244 (1994).

\section{Authors}

Zohre Arabi received her B.S. in computer engineering, software engineering, from payamnour University, Bonab Branch, Bonab, Iran, in 2008, the M.S. in computer engineering, computer rchitecture, from Islamic Azad University, Tabriz Branch, Tabriz, Iran, in 2010.From 2007, she worked as a researcher with the Young Researchers Club, Tabriz Branch, Islamic Azad University, Tabriz Branch. His research interests include Routing Protocol, Trust Grid Computing, and Wireless Networks.

Yaghoiub khodaei received her B.S. in computer engineering, software engineering, from payamnour University, Bonab Branch, Bonab, Iran, in 2009, he worker as a Jobholder with theBank Meli Iran, Tabriz, Iran , in 2005. 